\title{
Wireless Substitution: Early Release of Estimates From the National Health Interview Survey, July-December 2016
}

\author{
Stephen J. Blumberg, Ph.D., and Julian V. Luke \\ Division of Health Interview Statistics, National Center for Health Statistics
}

\section{Overview}

The second 6 months of 2016 was the first time that a majority of American homes had only wireless telephones. Preliminary results from the JulyDecember 2016 National Health Interview Survey (NHIS) indicate that $50.8 \%$ of American homes did not have a landline telephone but did have at least one wireless telephone (also known as cellular telephones, cell phones, or mobile phones) -an increase of 2.5 percentage points since the second 6 months of 2015. More than $70 \%$ of all adults aged $25-34$ and of adults renting their homes were living in wireless-only households. This report presents the most up-to-date estimates available from the federal government concerning the size and characteristics of this population.

\section{NHIS Early Release Program}

This report is published as part of the NHIS Early Release Program. Twice each year, the National Center for Health Statistics (NCHS) releases selected estimates of telephone coverage for the civilian noninstitutionalized U.S. population based on data from NHIS, along with comparable estimates from NHIS for the previous 3 years. The estimates are based on in-person interviews that are conducted throughout the year to collect information on health status, health-related behaviors, and health care access and utilization. The survey also includes information about household telephones and whether anyone in the household has a wireless telephone.
To provide access to the most recent information from NHIS, estimates using the July-December 2016 data are being released prior to final data editing and final weighting. These estimates should be considered preliminary. Estimates produced using the final data files may differ slightly from those presented here.

\section{Methods}

For many years, NHIS has asked respondents to provide residential telephone numbers, to permit the recontacting of survey participants. Starting in 2003, additional questions were asked to determine whether a family had a landline telephone. An NHIS family was considered to have landline telephone service if the survey respondent for the family reported that there was "at least one phone inside your home that is currently working and is not a cell phone." (To avoid possible confusion with cordless landline telephones, the word "wireless" was not used in the survey.)

An NHIS "family" is an individual or a group of two or more related persons living together in the same housing unit (a "household"). Thus, a family can consist of only one person, and more than one family can live in a household (including, for example, a household where there are multiple single-person families, as when unrelated roommates are living together).

The survey respondent for each family was also asked whether "anyone in your family has a working cellular telephone." Families are identified as "wireless families" if respondents reported that someone in the family had a working cell phone at the time of interview. This person (or persons) could be a civilian adult, a member of the military, or a child.

\section{Figure. Percentages of adults and children living in households with only wireless telephone service: United States, 2003-2016}

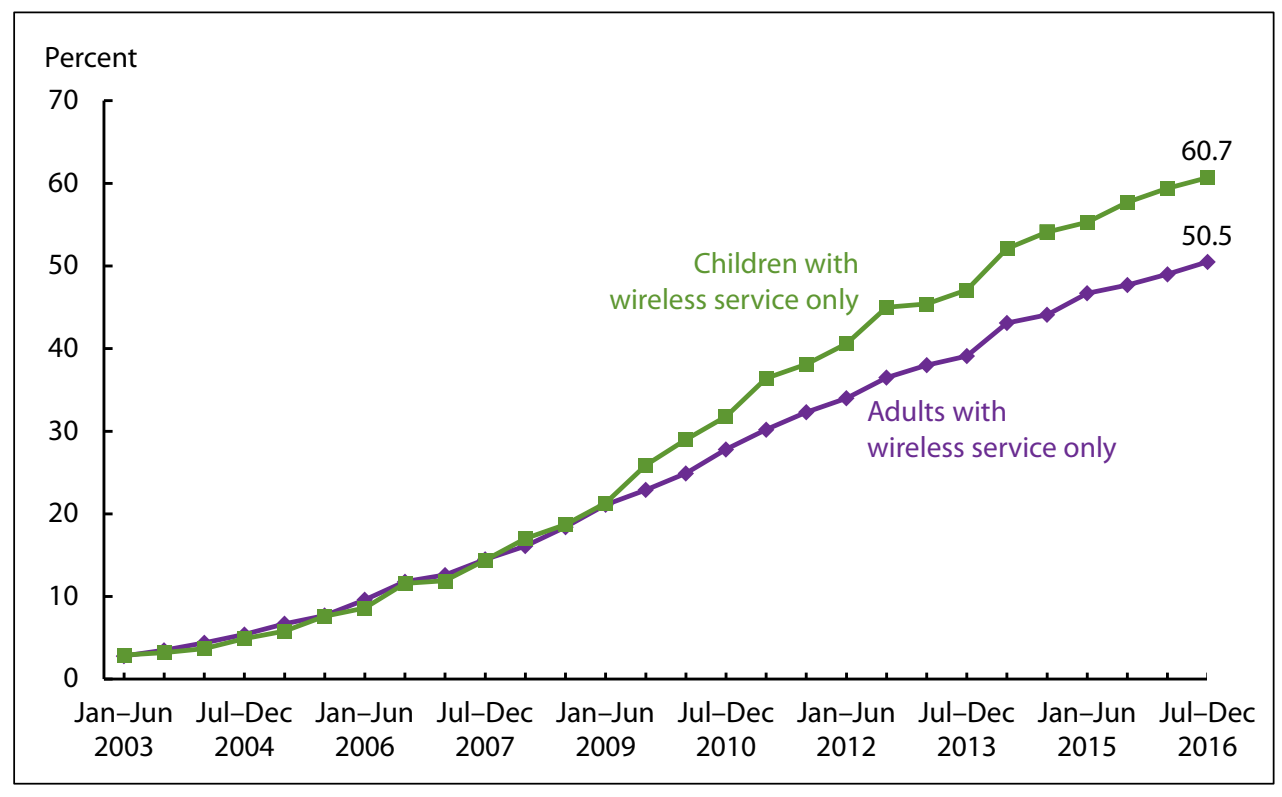

NOTE: Adults are aged 18 and over; children are under age 18.

DATA SOURCE: NCHS, National Health Interview Survey. 
Households are identified as "wireless-only" if they include at least one wireless family and if there are no families with landline telephone service in the household. Persons are identified as wireless-only if they live in a wireless-only household. A similar approach is used to identify adults living in households with no telephone service (neither wireless nor landline). Household telephone status (rather than family telephone status) is used in this report because most telephone surveys do not attempt to distinguish among families when more than one family lives in the same household.

From July through December 2016, information on household telephone status was obtained for 19,956 households that included at least one civilian adult or child. These households included 36,828 civilian adults aged 18 and over, and 11,437 children under age 18. Analyses of telephone status are presented separately for households, adults, and children in Table $\mathbf{1 .}$

Analyses of demographic characteristics are based on data from the NHIS Person and Household Files. Demographic data for all civilian adults living in interviewed households were used in these analyses. "Household income" is the sum of the family incomes in the household. Estimates stratified by household poverty status are based on reported income only because imputed income values are not available until a few months after the annual release of NHIS microdata. Household poverty status was unknown for $22.7 \%$ of adults in these analyses.

Analyses of selected health measures are based on data from the NHIS Sample Adult File. Health-related data for one randomly selected civilian adult in each family (the "sample adult") were used in these analyses. From July through December 2016, data on household telephone status and selected health measures were collected from 16,522 of these sample adults.

Because NHIS is conducted throughout the year and the sample is designed to yield a nationally representative sample each month, data can be analyzed quarterly. Weights are created for each calendar quarter of the NHIS sample. NHIS data weighting procedures are described in more detail in a previous NCHS report (Parsons et al., 2014).

Point estimates and 95\% confidence intervals were calculated using SUDAAN software (RTI International, Research Triangle Park, NC) to account for the complex sample design of NHIS.

Differences between percentages were evaluated using two-sided significance tests at the 0.05 level. Terms such as "more likely" and "less likely" indicate a statistically significant difference. Lack of comment regarding the difference between any two estimates does not necessarily mean that the difference was tested and found to be not significant. Because of small sample sizes, estimates based on less than 1 year of data may have large variances, and caution should be used in interpreting such estimates.

A new sample design was implemented with the 2016 NHIS. Sample areas were reselected to take account of changes in the distribution of the U.S. population since 2006, when the previous sample design was first implemented; commercial address lists were used as the main source of addresses, rather than field listing; and the oversampling procedures for black, Hispanic, and Asian persons that were a feature of the previous sample design were not implemented in 2016. Some differences between estimates for 2016 and estimates for earlier years may be attributable to the new sample design.

\section{Telephone Status}

In the second 6 months of 2016, more than one-half of all households (50.8\%) did not have a landline telephone but did have at least one wireless telephone (Table 1). More than 123 million adults (50.5\% of all adults) lived in households with only wireless telephones; over 44 million children (60.7\% of all children) lived in households with only wireless telephones.

The percentage of households that are wireless-only and the percentages of adults and children living in wireless-only households have been steadily increasing. The observed 2.5-percentage-point increase in the percentage of households that are wireless-only from the second 6 months of 2015 through the second 6 months of 2016 was statistically significant. The 2.8-percentage-point increase for adults and the 3.0percentage-point increase for children across the same 12-month time period were also significant (Figure). However, the increases from the first 6 months of 2016 to the second 6 months of 2016 were not statistically significant for adults $(p=0.11)$ or children $(p=0.36)$.

Approximately $3.2 \%$ of households had no telephone service (neither wireless nor landline) in the second 6 months of 2016. About 7.4 million adults (3.0\%) and 2.3 million children (3.1\%) lived in these households. The percentage of adults living without any telephone service has increased slightly but significantly over the past 3 years (Table 1). The corresponding percentage of children has not changed significantly $(p=.16)$.

\section{Demographic Differences}

The percentage of U.S. civilian noninstitutionalized adults living in wireless-only households is shown, by selected demographic characteristics and survey time period, in Table 2. For JulyDecember 2016:

- More than seven in ten adults aged 25-29 (72.7\%) and aged 30-34 (71.0\%) lived in households with only wireless telephones. These rates are greater than the rate for those 18-24 $(61.7 \%)$. The percentage of adults living with only wireless telephones decreased as age increased beyond 35 years: $62.5 \%$ for those $35-44 ; 45.2 \%$ for those 45-64; and $23.5 \%$ for those 65 and over.

- More than four in five adults living only with unrelated adult roommates (83.7\%) were in households with only wireless telephones. This rate is higher than the rates for adults living alone (54.7\%), adults living only with spouses or other adult family members (42.7\%), and adults living with children (58.1\%).

- More than seven in ten adults living in rented homes (71.5\%) had only wireless telephones. This rate is significantly higher than the rate for 
adults living in homes owned by a household member (40.9\%).

- Adults living in poverty $(66.3 \%)$ and near poverty (59.0\%) were more likely than higher income adults $(48.5 \%)$ to be living in households with only wireless telephones. (Footnote 3 in Table 2 gives definitions of these categories.)

- Hispanic adults (64.8\%) were more likely than non-Hispanic white (46.6\%), non-Hispanic black (52.1\%), or non-Hispanic Asian (47.4\%) adults to be living in households with only wireless telephones.

Geographic differences were also noted. Adults living in the Midwest (53.0\%), South (55.5\%), and West (53.4\%) were more likely than those living in the Northeast (34.2\%) to be living in households with only wireless telephones. Adults living in metropolitan areas (53.0\%) were more likely than those living in nonmetropolitan areas (47.0\%) to be living in wireless-only households.

\section{Demographic Distributions}

The demographic differences noted in the previous section are based on the distribution of household telephone status within each demographic group. When examining the population of wireless-only adults, some readers may instead wish to consider the distribution of various demographic characteristics within the wireless-only adult population.

Table 3 gives the percent distributions of selected demographic characteristics for adults living in households with only wireless telephones, by survey time period. The estimates in this table reveal that the distributions of selected demographic characteristics changed little over the 3-year period shown. The exceptions were related to age and home ownership status.

- The proportion of wireless-only adults who were aged 45 and over has increased steadily, from $34.2 \%$ in the second 6 months of 2013 to $39.7 \%$ in the second 6 months of 2016.
- The proportion of wireless-only adults living in homes owned by a household member increased from $48.5 \%$ in the second 6 months of 2013 to $54.4 \%$ in the second 6 months of 2016.

\section{Selected Health Measures by Household Telephone Status}

Many health surveys, political polls, and other types of research are conducted using random-digit-dial (RDD) telephone surveys. Despite operational challenges, most major survey research organizations include wireless telephone numbers when conducting RDD surveys. If they did not, the exclusion of households with only wireless telephones (along with the small proportion of households that have no telephone service) could bias results. This bias-known as coverage bias-could exist if there are differences between persons with and without landline telephones for the substantive variables of interest.

The NHIS Early Release Program updates and releases estimates for 15 key health indicators every 3 months. Table 4 presents estimates by household telephone status (landline, wireless-only, or phoneless) for all but two of these measures. ("Pneumococcal vaccination" and "personal care needs" were not included because these indicators are limited to older adults aged 65 and over.) For July-December 2016:

- Regarding alcohol consumption, the percentage of adults who had at least one heavy drinking day in the past year was substantially higher among wireless-only adults (29.8\%) than among adults living in landline households (18.8\%). Wireless-only adults were also more likely to be current smokers.

- Compared with adults living in landline households, wireless-only adults were more likely to have their health status described as excellent or very good, more likely to have met the 2008 federal physical activity guidelines for aerobic activity (based on leisure-time activity), and less likely to have ever been diagnosed with diabetes.

- The percentage without health insurance coverage at the time of interview among wireless-only adults under age 65 (13.6\%) was greater than the percentage among adults in that age group living in landline households (7.7\%).

- Compared with adults living in landline households, wireless-only adults were more likely to have experienced financial barriers to obtaining needed health care, and they were less likely to have a usual place to go for medical care. Wirelessonly adults were also less likely to have received an influenza vaccination during the previous year

- Wireless-only adults (46.1\%) were more likely than adults living in landline households (36.9\%) to have ever been tested for human immunodeficiency virus (HIV), the virus that causes AIDS.

The potential for bias due to undercoverage remains a real threat to health surveys that do not include sufficient representation of households with only wireless telephones.

\section{Wireless-mostly Households}

The potential for bias due to undercoverage is not the only threat to surveys conducted only on landline telephones. Researchers are also concerned that some people living in households with landlines cannot be reached on those landlines because they rely on wireless telephones for all or almost all of their calls.

In 2007, a question was added to NHIS for persons living in families with both landline and cellular telephones. The respondent for the family was asked to consider all of the telephone calls his or her family receives and to report whether "all or almost all calls are received on cell phones, some are received on cell phones and some on regular phones, or very few or none are received on cell phones." This question permits the identification of persons living in "wireless-mostly" 
households-defined as households with both landline and cellular telephones in which all families receive all or almost all calls on cell phones.

Among households with both landline and wireless telephones, $38.0 \%$ received all or almost all calls on wireless telephones, based on data for JulyDecember 2016. These wireless-mostly households make up $15.0 \%$ of all households. During the second 6 months of 2016, about 41 million adults (16.7\%) lived in wireless-mostly households.

Table 5 gives the percentage of adults living in wireless-mostly households, by demographic characteristics and survey time period. For July-December 2016:

- Adults with college degrees (19.6\%) were more likely to be living in wireless-mostly households than were high school graduates (14.8\%) or adults with less education (12.2\%).

- Adults living with children (19.4\%) were more likely than adults living alone $(9.9 \%)$ to be living in wirelessmostly households.

- Adults living in poverty (10.0\%) and adults living near poverty (11.1\%) were less likely than higher-income adults (18.9\%) to be living in wirelessmostly households.

- Adults living in rented homes (10.5\%) were less likely to be living in wireless-mostly households than were adults living in homes owned by a household member (19.7\%).

NHIS data cannot be used to estimate the proportion of wirelessmostly adults who are unreachable or to estimate the potential for bias due to their exclusion from landline surveys.

\section{State Estimates}

The potential for bias may differ from one state to another because the prevalence of wireless-only households varies substantially across states. For more information about prevalence estimates at the state level, see

- NCHS. Modeled estimates (with standard errors) of the percent distribution of household telephone status for adults aged 18 and over, by state: United States, 2015. August 2016. Available from: http://www.cdc.gov/nchs/data/nhis/ earlyrelease/wireless_state_201608.p df.

- Blumberg SJ, Ganesh N, Luke JV, Gonzales G. Wireless substitution: State-level estimates from the National Health Interview Survey, 2012. National health statistics reports; no 70. Hyattsville, MD: National Center for Health Statistics. 2013. Available from:

http://www.cdc.gov/nchs/data/nhsr/ nhsr070.pdf.

\section{Other NHIS Early Release Program Products}

Two additional reports are published regularly as part of the NHIS Early Release Program. Early Release of Selected Estimates Based on Data From the National Health

Interview Survey is published quarterly and provides estimates for 15 selected measures of health. Health Insurance Coverage: Early Release of Estimates From the National Health Interview Survey is also published quarterly and provides additional estimates regarding health insurance coverage. Other Early Release Program products are released as needed. In addition to these reports, preliminary microdata files containing selected NHIS variables are produced as part of the ER Program. Beginning in May 2016, the telephone service use variables presented in this report have been included in those microdata files. These variables are made available twice each year, in November or December for data from the first 6 months of the calendar year and in May or June for data from the second 6 months of the calendar year. NHIS data users can analyze these files through the NCHS Research Data Centers (http://www.cdc.gov/rdc/) without having to wait for the final annual NHIS microdata files to be released.

For more information about NHIS and the NHIS Early Release Program, or to find other Early Release Program products, see
- $\quad$ NHIS home page at http://www.cdc.gov/nchs/nhis.htm.

- Early Release Program home page at http://www.cdc.gov/nchs/nhis/ releases.htm.

- Parsons VL, Moriarity CL, Jonas K, et al. Design and estimation for the National Health Interview Survey: 2006-2015. National Center for Health Statistics. Vital Health Stat 2(165). 2014. Available from: http://www.cdc.gov/nchs/data/ series/sr_02/sr02_165.pdf.

\section{Suggested Citation}

Blumberg SJ, Luke JV. Wireless substitution: Early release of estimates from the National Health Interview Survey, July-December 2016. National Center for Health Statistics. May 2017. Available from: http://www.cdc.gov/nchs/nhis.htm. 
Wireless Substitution: Early Release of Estimates From the National Health Interview Survey, July-December 2016

Table 1. Percent distribution of household telephone status for households, adults, and children, by date of interview: United States, July $2013-D e c e m b e r ~ 2016$

\begin{tabular}{|c|c|c|c|c|c|c|c|c|}
\hline Date of interview & $\begin{array}{c}\text { Number of } \\
\text { households } \\
\text { (unweighted) }\end{array}$ & $\begin{array}{l}\text { Landline with } \\
\text { wireless }\end{array}$ & $\begin{array}{l}\text { Landline without } \\
\text { wireless }\end{array}$ & $\begin{array}{l}\text { Landline with } \\
\text { unknown wireless }\end{array}$ & $\begin{array}{l}\text { Nonlandline with } \\
\text { unknown wireless }\end{array}$ & Wireless-only & Phoneless & Total \\
\hline \multicolumn{9}{|l|}{ Households } \\
\hline July-December 2013 & 21,512 & 47.7 & 8.6 & 0.1 & 0.1 & 41.0 & 2.5 & 100.0 \\
\hline January-June 2014 & 22,438 & 44.7 & 8.5 & 0.1 & 0.0 & 44.0 & 2.6 & 100.0 \\
\hline July-December 2014 & 22,023 & 42.7 & 8.4 & 0.2 & 0.1 & 45.4 & 3.2 & 100.0 \\
\hline January-June 2015 & 21,517 & 41.6 & 7.6 & 0.1 & 0.0 & 47.4 & 3.4 & 100.0 \\
\hline July-December 2015 & 19,959 & 41.2 & 7.2 & 0.1 & 0.0 & 48.3 & 3.1 & 100.0 \\
\hline January-June 2016 & 20,206 & 40.2 & 7.2 & 0.1 & 0.0 & 49.3 & 3.1 & 100.0 \\
\hline July-December 2016 & 19,956 & 39.4 & 6.5 & 0.0 & 0.0 & 50.8 & 3.2 & 100.0 \\
\hline $95 \%$ confidence interval ${ }^{1}$ & $\ldots$ & $38.44-40.34$ & $6.09-7.03$ & $0.02-0.08$ & $0.02-0.09$ & $49.76-51.76$ & $2.92-3.56$ & $\cdots$ \\
\hline \multicolumn{9}{|l|}{ Adults } \\
\hline July-December 2013 & 40,173 & 51.5 & 7.0 & 0.1 & 0.1 & 39.1 & 2.2 & 100.0 \\
\hline January-June 2014 & 42,262 & 47.3 & 7.0 & 0.1 & 0.1 & 43.1 & 2.4 & 100.0 \\
\hline July-December 2014 & 41,160 & 45.8 & 7.1 & 0.1 & 0.1 & 44.1 & 2.9 & 100.0 \\
\hline January-June 2015 & 40,489 & 43.9 & 6.2 & 0.1 & 0.0 & 46.7 & 3.1 & 100.0 \\
\hline July-December 2015 & 37,332 & 43.7 & 5.8 & 0.1 & 0.0 & 47.7 & 2.7 & 100.0 \\
\hline January-June 2016 & 36,885 & 42.1 & 5.8 & 0.1 & 0.0 & 49.0 & 2.9 & 100.0 \\
\hline July-December 2016 & 36,828 & 41.0 & 5.4 & 0.0 & 0.0 & 50.5 & 3.0 & 100.0 \\
\hline $95 \%$ confidence interval ${ }^{1}$ & $\ldots$ & $39.92-42.16$ & $4.96-5.79$ & $0.02-0.08$ & $0.02-0.11$ & $49.27-51.72$ & $2.72-3.35$ & $\ldots$ \\
\hline \multicolumn{9}{|l|}{ Children } \\
\hline July-December 2013 & 13,714 & 46.4 & 3.8 & 0.1 & 0.0 & 47.1 & 2.5 & 100.0 \\
\hline January-June 2014 & 14,349 & 41.7 & 3.5 & - & 0.0 & 52.1 & 2.7 & 100.0 \\
\hline July-December 2014 & 13,754 & 39.1 & 3.3 & 0.1 & 0.0 & 54.1 & 3.4 & 100.0 \\
\hline January-June 2015 & 13,493 & 38.3 & 3.0 & 0.1 & 0.0 & 55.3 & 3.2 & 100.0 \\
\hline July-December 2015 & 12,197 & 36.2 & 2.8 & 0.1 & 0.0 & 57.7 & 3.1 & 100.0 \\
\hline January-June 2016 & 11,552 & 34.6 & 2.5 & 0.1 & 0.0 & 59.4 & 3.4 & 100.0 \\
\hline July-December 2016 & 11,437 & 33.5 & 2.6 & 0.0 & 0.1 & 60.7 & 3.1 & 100.0 \\
\hline $95 \%$ confidence interval ${ }^{1}$ & $\ldots$ & $31.72-35.29$ & $2.11-3.14$ & $0.00-0.16$ & $0.04-0.32$ & $58.70-62.64$ & $2.61-3.73$ & $\ldots$ \\
\hline
\end{tabular}

0.0 Quantity more than zero but less than 0.05 .

... Category not applicable.

- Quantity zero.

'Refers to July-December 2016.

NOTE: Data are based on household interviews of a sample of the civilian noninstitutionalized population.

DATA SOURCE: NCHS, National Health Interview Survey, July 2013-December 2016. 
Wireless Substitution: Early Release of Estimates From the National Health Interview Survey, July-December 2016

Table 2. Percentage of adults living in wireless-only households, by selected demographic characteristics and calendar half-years: United States, July $2013-$ December 2016

\begin{tabular}{|c|c|c|c|c|c|c|c|c|}
\hline Demographic characteristic & $\begin{array}{c}\text { July-December } \\
2013\end{array}$ & $\begin{array}{c}\text { January-June } \\
2014\end{array}$ & $\begin{array}{l}\text { July-December } \\
2014\end{array}$ & $\begin{array}{c}\text { January-June } \\
2015\end{array}$ & $\begin{array}{l}\text { July-December } \\
2015\end{array}$ & $\begin{array}{c}\text { January-June } \\
2016\end{array}$ & $\begin{array}{l}\text { July-December } \\
2016\end{array}$ & $\begin{array}{l}95 \% \text { confidence } \\
\text { interval }^{1}\end{array}$ \\
\hline \multicolumn{9}{|l|}{ Race/ethnicity } \\
\hline Hispanic or Latino, any race(s) & 53.1 & 56.1 & 58.6 & 59.2 & 60.5 & 63.7 & 64.8 & $61.94-67.47$ \\
\hline Non-Hispanic white, single race & 35.1 & 39.6 & 40.3 & 43.2 & 44.0 & 45.0 & 46.6 & $45.32-47.89$ \\
\hline Non-Hispanic black, single race & 42.7 & 44.9 & 45.7 & 48.1 & 48.5 & 49.2 & 52.1 & $49.23-54.98$ \\
\hline Non-Hispanic Asian, single race & 38.1 & 41.3 & 42.3 & 47.9 & 48.4 & 51.4 & 47.4 & $42.18-52.61$ \\
\hline Non-Hispanic other, single race & 51.7 & 52.4 & 54.8 & 51.8 & 56.5 & 57.5 & 57.9 & $50.25-65.12$ \\
\hline Non-Hispanic multiple race & 45.7 & 52.5 & 53.3 & 53.6 & 60.2 & 53.9 & 62.2 & $56.97-67.24$ \\
\hline \multicolumn{9}{|l|}{ Age (years) } \\
\hline $18-24$ & 53.0 & 57.8 & 58.0 & 59.4 & 61.1 & 62.7 & 61.7 & $59.23-64.03$ \\
\hline $25-29$ & 65.7 & 69.3 & 69.2 & 71.3 & 72.6 & 72.1 & 72.7 & $71.01-74.37$ \\
\hline $30-34$ & 59.7 & 64.9 & 67.4 & 67.8 & 69.0 & 69.8 & 71.0 & $68.79-73.14$ \\
\hline $35-44$ & 47.8 & 52.5 & 53.7 & 56.6 & 58.2 & 60.0 & 62.5 & $60.38-64.60$ \\
\hline $45-64$ & 31.4 & 35.7 & 36.8 & 40.8 & 41.2 & 43.3 & 45.2 & $43.88-46.57$ \\
\hline 65 and over & 13.6 & 15.7 & 17.1 & 19.3 & 20.5 & 21.1 & 23.5 & $22.19-24.86$ \\
\hline \multicolumn{9}{|l|}{ Sex } \\
\hline Male & 40.4 & 44.3 & 45.7 & 48.2 & 49.3 & 50.3 & 51.6 & $50.30-52.93$ \\
\hline Female & 37.9 & 41.9 & 42.6 & 45.3 & 46.1 & 47.8 & 49.4 & $48.24-50.66$ \\
\hline \multicolumn{9}{|l|}{ Education } \\
\hline Some high school or less & 41.8 & 46.6 & 46.5 & 49.0 & 51.1 & 52.1 & 55.2 & $52.86-57.54$ \\
\hline High school graduate or GED 2 & 38.8 & 43.3 & 44.2 & 46.7 & 47.2 & 48.4 & 50.2 & $48.60-51.89$ \\
\hline Some post-high school, no degree & 41.7 & 45.6 & 47.1 & 49.0 & 49.7 & 50.8 & 52.4 & $51.05-53.74$ \\
\hline 4-year college degree or higher & 35.5 & 39.0 & 40.3 & 43.5 & 44.8 & 46.5 & 47.1 & $45.08-49.05$ \\
\hline \multicolumn{9}{|l|}{ Employment status last week } \\
\hline Working at a job or business & 44.4 & 48.9 & 49.9 & 52.7 & 53.7 & 55.6 & 56.4 & $55.03-57.67$ \\
\hline Keeping house & 40.5 & 47.6 & 47.2 & 49.3 & 50.7 & 53.0 & 54.9 & $51.47-58.34$ \\
\hline Going to school & 46.3 & 49.7 & 53.8 & 49.6 & 53.2 & 53.4 & 58.9 & $54.95-62.75$ \\
\hline Something else (incl. unemployed) & 27.0 & 29.1 & 29.7 & 32.7 & 33.4 & 33.5 & 35.7 & $34.37-37.09$ \\
\hline \multicolumn{9}{|l|}{ Household structure } \\
\hline Adult living alone & 46.6 & 48.3 & 49.5 & 51.1 & 52.1 & 53.3 & 54.7 & $53.47-55.88$ \\
\hline Unrelated adults, no children & 76.1 & 73.9 & 81.3 & 84.6 & 78.8 & 79.1 & 83.7 & $77.80-88.29$ \\
\hline Related adults, no children & 31.0 & 35.3 & 35.8 & 39.1 & 39.7 & 40.7 & 42.7 & $41.24-44.18$ \\
\hline Adult(s) with children & 44.8 & 49.8 & 50.8 & 53.3 & 55.2 & 57.0 & 58.1 & $55.99-60.11$ \\
\hline \multicolumn{9}{|l|}{ Household poverty status ${ }^{3}$} \\
\hline Poor & 56.2 & 59.1 & 59.4 & 59.3 & 64.3 & 63.1 & 66.3 & $63.44-69.02$ \\
\hline Near-poor & 46.1 & 50.8 & 51.1 & 54.4 & 54.0 & 54.0 & 59.0 & $56.40-61.57$ \\
\hline Not-poor & 36.6 & 40.8 & 42.5 & 45.7 & 45.7 & 48.2 & 48.5 & $46.87-50.15$ \\
\hline
\end{tabular}

See footnotes at end of table. 
Wireless Substitution: Early Release of Estimates From the National Health Interview Survey, July-December 2016

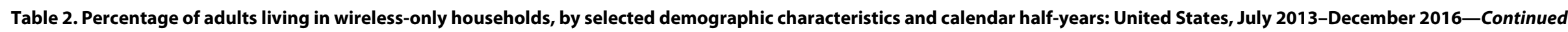

\begin{tabular}{|c|c|c|c|c|c|c|c|c|}
\hline Demographic characteristic & $\begin{array}{l}\text { July-December } \\
2013\end{array}$ & $\begin{array}{l}\text { January-June } \\
2014\end{array}$ & $\begin{array}{l}\text { July-December } \\
2014\end{array}$ & $\begin{array}{l}\text { January-June } \\
2015\end{array}$ & $\begin{array}{l}\text { July-December } \\
\quad 2015\end{array}$ & $\begin{array}{l}\text { January-June } \\
2016\end{array}$ & $\begin{array}{l}\text { July-December } \\
2016\end{array}$ & $\begin{array}{l}\text { 95\% confidence } \\
\text { interval }^{1}\end{array}$ \\
\hline \multicolumn{9}{|l|}{ Geographic region ${ }^{4}$} \\
\hline Northeast & 24.9 & 27.8 & 29.5 & 31.6 & 31.4 & 32.4 & 34.2 & $31.15-37.33$ \\
\hline Midwest & 43.7 & 46.9 & 48.0 & 51.9 & 51.4 & 51.7 & 53.0 & $50.98-54.99$ \\
\hline South & 41.9 & 47.3 & 47.0 & 50.2 & 51.3 & 52.3 & 55.4 & $53.45-57.40$ \\
\hline West & 41.2 & 43.8 & 46.9 & 47.1 & 51.2 & 54.4 & 53.4 & $50.94-55.82$ \\
\hline \multicolumn{9}{|l|}{ Metropolitan statistical area status } \\
\hline Metropolitan & 40.5 & 43.9 & 45.7 & 47.8 & 48.4 & 51.6 & 53.0 & $51.67-54.36$ \\
\hline Not metropolitan & 33.7 & 39.8 & 37.6 & 42.3 & 43.1 & 46.3 & 47.0 & $44.38-49.57$ \\
\hline \multicolumn{9}{|l|}{ Home ownership status ${ }^{5}$} \\
\hline Owned or being bought & 28.5 & 32.9 & 33.1 & 37.2 & 37.3 & 39.0 & 40.9 & $39.54-42.21$ \\
\hline Renting & 61.7 & 64.6 & 66.2 & 67.0 & 68.8 & 69.7 & 71.5 & $70.02-72.87$ \\
\hline Other arrangement & 49.3 & 52.2 & 49.2 & 52.8 & 58.0 & 52.0 & 53.9 & $47.71-60.03$ \\
\hline $\begin{array}{l}\text { Number of wireless-only adults in } \\
\text { survey sample (unweighted) }\end{array}$ & 16,436 & 18,380 & 18,740 & 18,921 & 17,974 & 17,896 & 18,387 & $\ldots$ \\
\hline
\end{tabular}

\footnotetext{
... Category not applicable.
}

'Refers to July-December 2016

${ }^{2} \mathrm{GED}$ is General Educational Development high school equivalency diploma.

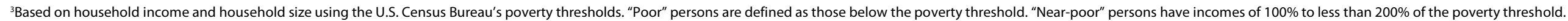

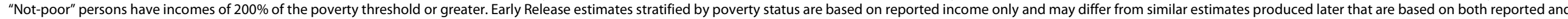

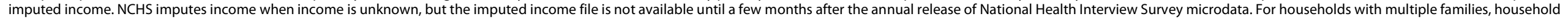
income and household size were calculated as the sum of the multiple measures of family income and family size.

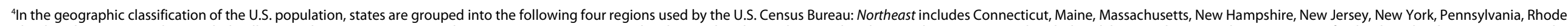

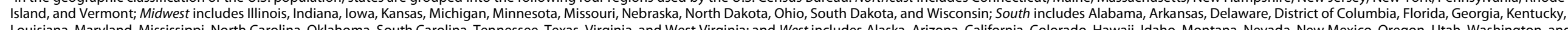

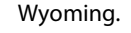

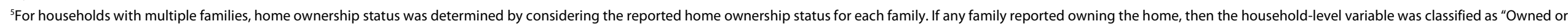

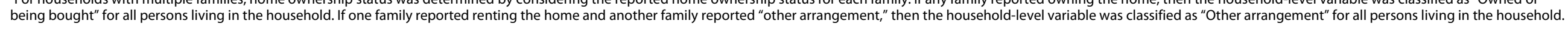
NOTE: Data are based on household interviews of a sample of the civilian noninstitutionalized population.

DATA SOURCE: CDC/NCHS, National Health Interview Survey, July 2013-December 2016. 
Wireless Substitution: Early Release of Estimates From the National Health Interview Survey, July-December 2016

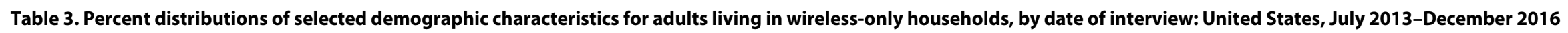

\begin{tabular}{|c|c|c|c|c|c|c|c|c|}
\hline Demographic characteristic & $\begin{array}{l}\text { July-December } \\
2013\end{array}$ & $\begin{array}{c}\text { January-June } \\
2014\end{array}$ & $\begin{array}{l}\text { July-December } \\
2014\end{array}$ & $\begin{array}{c}\text { January-June } \\
2015\end{array}$ & $\begin{array}{l}\text { July-December } \\
2015\end{array}$ & $\begin{array}{c}\text { January-June } \\
2016\end{array}$ & $\begin{array}{l}\text { July-December } \\
2016\end{array}$ & $\begin{array}{c}95 \% \text { confidence } \\
\text { interval }^{1}\end{array}$ \\
\hline \multicolumn{9}{|l|}{ Race/ethnicity } \\
\hline Hispanic or Latino, any race(s) & 20.5 & 19.8 & 20.3 & 19.6 & 19.9 & 20.5 & 20.3 & $18.32-22.48$ \\
\hline Non-Hispanic white, single race & 59.2 & 60.4 & 60.0 & 60.2 & 59.7 & 59.1 & 59.3 & $56.77-61.76$ \\
\hline Non-Hispanic black, single race & 12.6 & 12.1 & 12.1 & 12.1 & 12.0 & 11.7 & 12.2 & $10.96-13.57$ \\
\hline Non-Hispanic Asian, single race & 5.2 & 5.2 & 5.3 & 5.8 & 5.8 & 6.1 & 5.5 & $4.44-6.72$ \\
\hline Non-Hispanic other, single race & 1.0 & 0.9 & 0.7 & 0.8 & 1.0 & 0.9 & 0.9 & $0.73-1.11$ \\
\hline Non-Hispanic multiple race & 1.4 & 1.6 & 1.5 & 1.5 & 1.7 & 1.8 & 1.8 & $1.59-2.06$ \\
\hline Total & 100.0 & 100.0 & 100.0 & 100.0 & 100.0 & 100.0 & 100.0 & $\ldots$ \\
\hline \multicolumn{9}{|l|}{ Age (years) } \\
\hline $18-24$ & 17.4 & 17.1 & 16.6 & 16.0 & 16.0 & 15.8 & 14.9 & $14.20-15.59$ \\
\hline $25-29$ & 14.8 & 14.1 & 13.9 & 13.6 & 13.6 & 13.3 & 13.0 & $12.40-13.53$ \\
\hline $30-34$ & 13.3 & 13.1 & 13.2 & 12.6 & 12.6 & 12.4 & 12.3 & $11.73-12.90$ \\
\hline $35-44$ & 20.4 & 20.3 & 20.1 & 19.9 & 20.0 & 19.9 & 20.2 & 19.39-21.03 \\
\hline $45-64$ & 27.8 & 28.6 & 28.8 & 30.0 & 29.6 & 30.2 & 30.5 & 29.62-31.39 \\
\hline 65 and over & 6.4 & 6.8 & 7.3 & 7.9 & 8.3 & 8.4 & 9.2 & $8.68-9.69$ \\
\hline Total & 100.0 & 100.0 & 100.0 & 100.0 & 100.0 & 100.0 & 100.0 & $\cdots$ \\
\hline \multicolumn{9}{|l|}{ Sex } \\
\hline Male & 49.7 & 49.6 & 49.9 & 49.8 & 49.9 & 49.5 & 49.3 & $48.81-49.78$ \\
\hline Female & 50.3 & 50.4 & 50.1 & 50.2 & 50.1 & 50.5 & 50.7 & $50.22-51.19$ \\
\hline Total & 100.0 & 100.0 & 100.0 & 100.0 & 100.0 & 100.0 & 100.0 & $\ldots$ \\
\hline \multicolumn{9}{|l|}{ Education } \\
\hline Some high school or less & 14.5 & 14.7 & 13.9 & 13.5 & 13.6 & 14.2 & 12.9 & $12.11-13.70$ \\
\hline High school graduate or GED ${ }^{2}$ & 26.9 & 27.2 & 26.9 & 26.0 & 25.8 & 26.3 & 25.8 & $24.72-26.97$ \\
\hline Some post-high school, no degree & 32.4 & 32.2 & 31.9 & 32.0 & 31.7 & 30.9 & 32.3 & $31.29-33.26$ \\
\hline 4-year college degree or higher & 26.2 & 25.9 & 27.3 & 28.5 & 28.9 & 28.7 & 29.0 & $27.87-30.20$ \\
\hline Total & 100.0 & 100.0 & 100.0 & 100.0 & 100.0 & 100.0 & 100.0 & $\ldots$ \\
\hline \multicolumn{9}{|l|}{ Employment status last week } \\
\hline Working at a job or business & 70.1 & 69.3 & 70.1 & 69.7 & 69.7 & 70.3 & 69.9 & $69.01-70.80$ \\
\hline Keeping house & 5.7 & 6.4 & 6.0 & 6.0 & 5.9 & 5.8 & 5.7 & $5.23-6.29$ \\
\hline Going to school & 3.6 & 4.1 & 4.0 & 3.6 & 3.7 & 3.4 & 3.6 & $3.26-3.96$ \\
\hline Something else (incl. unemployed) & 19.8 & 19.5 & 19.1 & 19.8 & 20.0 & 19.7 & 20.1 & $19.36-20.84$ \\
\hline Unknown, not reported & 0.8 & 0.9 & 0.9 & 0.8 & 0.7 & 0.8 & 0.7 & $0.41-1.04$ \\
\hline Total & 100.0 & 100.0 & 100.0 & 100.0 & 100.0 & 100.0 & 100.0 & $\ldots$ \\
\hline \multicolumn{9}{|l|}{ Household structure } \\
\hline Adult living alone & 18.6 & 17.0 & 17.5 & 16.4 & 17.4 & 17.0 & 16.2 & $15.58-16.88$ \\
\hline Unrelated adults, no children & 2.9 & 2.5 & 2.9 & 2.4 & 2.6 & 1.9 & 1.8 & $1.48-2.27$ \\
\hline Related adults, no children & 36.9 & 38.8 & 37.9 & 39.6 & 39.6 & 39.3 & 40.4 & $39.16-41.72$ \\
\hline Adult(s) with children & 41.6 & 41.8 & 41.6 & 41.6 & 40.4 & 41.8 & 41.5 & $40.16-42.88$ \\
\hline Total & 100.0 & 100.0 & 100.0 & 100.0 & 100.0 & 100.0 & 100.0 & $\ldots$ \\
\hline
\end{tabular}


Wireless Substitution: Early Release of Estimates From the National Health Interview Survey, July-December 2016

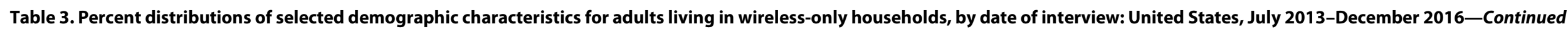

\begin{tabular}{|c|c|c|c|c|c|c|c|c|}
\hline Demographic characteristic & $\begin{array}{l}\text { July-December } \\
2013\end{array}$ & $\begin{array}{l}\text { January-June } \\
2014\end{array}$ & $\begin{array}{l}\text { July-December } \\
2014\end{array}$ & $\begin{array}{c}\text { January-June } \\
2015\end{array}$ & $\begin{array}{l}\text { July-December } \\
2015\end{array}$ & $\begin{array}{c}\text { January-June } \\
2016\end{array}$ & $\begin{array}{l}\text { July-December } \\
2016\end{array}$ & $\begin{array}{l}\text { 95\% confidence } \\
\text { interval }^{1}\end{array}$ \\
\hline \multicolumn{9}{|l|}{ Household poverty status ${ }^{3}$} \\
\hline Poor & 14.1 & 13.0 & 13.6 & 10.9 & 12.1 & 10.9 & 10.8 & $9.97-11.71$ \\
\hline Near-poor & 16.6 & 16.7 & 15.9 & 15.5 & 15.6 & 14.9 & 15.4 & $14.48-16.39$ \\
\hline Not-poor & 47.8 & 49.4 & 49.3 & 53.1 & 50.8 & 53.8 & 53.7 & $52.35-55.13$ \\
\hline Unknown, not reported & 21.5 & 20.8 & 21.3 & 20.5 & 21.5 & 20.4 & 20.0 & $18.78-21.33$ \\
\hline Total & 100.0 & 100.0 & 100.0 & 100.0 & 100.0 & 100.0 & 100.0 & $\ldots$ \\
\hline \multicolumn{9}{|l|}{ Geographic region ${ }^{4}$} \\
\hline Northeast & 11.3 & 11.1 & 12.0 & 11.5 & 12.1 & 12.1 & 12.5 & $11.00-14.15$ \\
\hline Midwest & 25.1 & 25.0 & 24.3 & 25.0 & 23.2 & 23.3 & 22.7 & $20.37-25.24$ \\
\hline South & 39.9 & 41.1 & 39.9 & 39.9 & 40.5 & 38.5 & 39.6 & $34.66-44.81$ \\
\hline West & 23.8 & 22.9 & 23.8 & 23.5 & 24.2 & 26.2 & 25.2 & $20.67-30.29$ \\
\hline Total & 100.0 & 100.0 & 100.0 & 100.0 & 100.0 & 100.0 & 100.0 & $\ldots$ \\
\hline \multicolumn{9}{|l|}{ Metropolitan statistical area status } \\
\hline Metropolitan & 82.6 & 81.6 & 83.1 & 82.3 & 87.8 & 83.8 & 84.1 & $81.51-86.38$ \\
\hline Not metropolitan & 17.4 & 18.4 & 16.9 & 17.7 & 12.2 & 16.2 & 15.9 & $13.62-18.49$ \\
\hline Total & 100.0 & 100.0 & 100.0 & 100.0 & 100.0 & 100.0 & 100.0 & $\ldots$ \\
\hline \multicolumn{9}{|l|}{ Home ownership status ${ }^{5}$} \\
\hline Owned or being bought & 48.5 & 51.1 & 49.5 & 53.8 & 51.6 & 52.9 & 54.4 & $53.03-55.83$ \\
\hline Renting & 49.1 & 46.4 & 48.4 & 44.2 & 45.8 & 45.1 & 43.4 & $41.88-44.84$ \\
\hline Other arrangement & 2.4 & 2.6 & 2.1 & 2.0 & 2.6 & 2.0 & 2.2 & $1.87-2.61$ \\
\hline Total & 100.0 & 100.0 & 100.0 & 100.0 & 100.0 & 100.0 & 100.0 & $\ldots$ \\
\hline $\begin{array}{l}\text { Number of wireless-only adults in } \\
\text { survey sample (unweighted) }\end{array}$ & 16,436 & 18,380 & 18,740 & 18,921 & 17,974 & 17,896 & 18,387 & $\ldots$ \\
\hline
\end{tabular}

\section{... Category not applicable.}

'Refers to July-December 2016.

${ }^{2}$ GED is General Educational Development high school equivalency diploma.

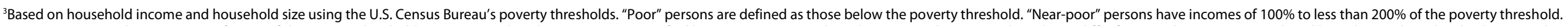

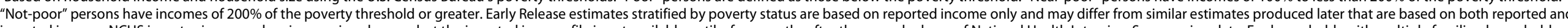

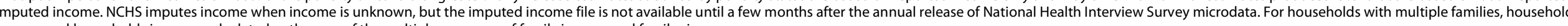

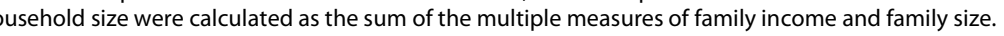

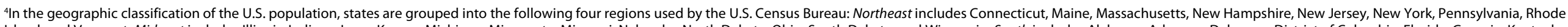

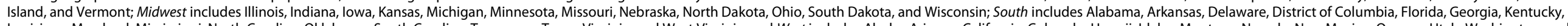

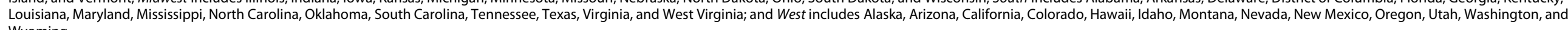
Wyoming.

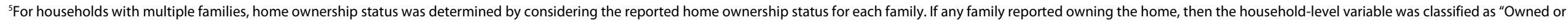

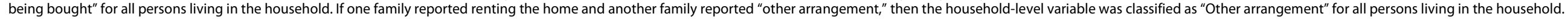
NOTE: Data are based on household interviews of a sample of the civilian noninstitutionalized population.

DATA SOURCE: CDC/NCHS, National Health Interview Survey, July 2013-December 2016. 
Wireless Substitution: Early Release of Estimates From the National Health Interview Survey, July-December 2016

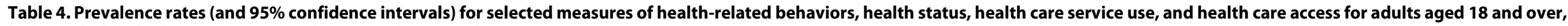
by household telephone status: United States, July-December 2016

\begin{tabular}{|c|c|c|c|}
\hline Measure & Landline $^{1}$ & Wireless-only & Phoneless \\
\hline \multicolumn{4}{|l|}{ Health-related behaviors } \\
\hline At least one heavy drinking day in past year ${ }^{2}$ & $18.8(17.58-20.06)$ & $29.8(28.29-31.32)$ & $24.5(20.06-29.63)$ \\
\hline Current smoker ${ }^{3}$ & $12.1(11.08-13.16)$ & $18.4(17.09-19.79)$ & $19.3(15.05-24.52)$ \\
\hline $\begin{array}{l}\text { Met the } 2008 \text { federal physical activity guidelines for aerobic activity through } \\
\text { leisure-time aerobic activity }{ }^{4}\end{array}$ & $36.9(35.52-38.35)$ & $41.5(39.99-43.01)$ & $35.7(31.04-40.66)$ \\
\hline \multicolumn{4}{|l|}{ Health status } \\
\hline Health status described as excellent or very good ${ }^{5}$ & $59.8(58.54-60.99)$ & $62.9(61.37-64.42)$ & $56.6(50.66-62.43)$ \\
\hline Experienced serious psychological distress in past 30 days $^{6}$ & $2.4(2.02-2.88)$ & $4.6(4.01-5.22)$ & $3.2(1.78-5.65)$ \\
\hline Obese (adults aged 20 and over) ${ }^{7}$ & $30.7(29.29-32.13)$ & $30.1(28.82-31.38)$ & $25.6(21.26-30.52)$ \\
\hline Asthma episode in past year ${ }^{8}$ & $3.9(3.36-4.49)$ & $4.1(3.53-4.70)$ & *2.6 (1.37-4.88) \\
\hline Ever diagnosed with diabetes ${ }^{9}$ & $12.2(11.28-13.15)$ & $7.3(6.64-8.06)$ & $8.6(5.60-12.93)$ \\
\hline \multicolumn{4}{|l|}{ Health care service use } \\
\hline Received influenza vaccine during past year ${ }^{10}$ & $49.7(48.27-51.09)$ & $35.2(33.94-36.51)$ & $37.4(31.82-43.38)$ \\
\hline Ever been tested for $\mathrm{HIV}^{11}$ & $36.9(35.27-38.55)$ & $46.1(44.53-47.73)$ & $38.7(32.93-44.77)$ \\
\hline \multicolumn{4}{|l|}{ Health care access } \\
\hline Has a usual place to go for medical care ${ }^{12}$ & $92.2(91.26-93.05)$ & $81.1(79.69-82.43)$ & $81.4(77.22-84.96)$ \\
\hline Failed to obtain needed medical care in past year due to financial barriers ${ }^{13}$ & $3.9(3.36-4.51)$ & $7.8(7.15-8.55)$ & $10.0(7.21-13.77)$ \\
\hline Currently uninsured (adults aged $18-64)^{14}$ & $7.7(6.58-8.89)$ & $13.6(12.30-15.08)$ & $19.5(14.32-26.00)$ \\
\hline Number of adults in survey sample (unweighted) & 7,422 & 8,607 & 493 \\
\hline
\end{tabular}

* Estimate has a relative standard error greater than $30 \%$ and does not meet standards for reliability or precision.

'Includes households that also have wireless telephone service.

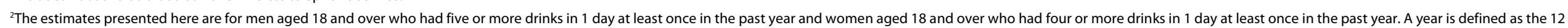
months prior to interview. The analyses excluded adults with unknown alcohol consumption (about 1\%).

${ }^{3} \mathrm{~A}$ person who had smoked more than 100 cigarettes in his or her lifetime and now smokes every day or some days. The analyses excluded adults with unknown smoking status (about 2\%).

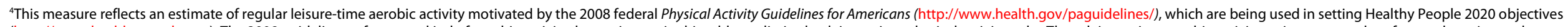

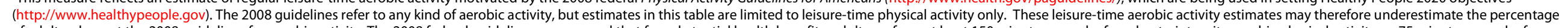

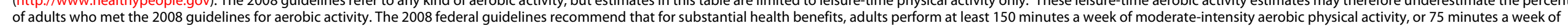

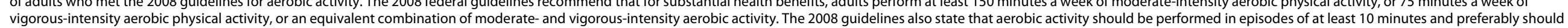
be spread throughout the week. The analyses excluded adults with unknown physical activity participation (about $3 \%$ ).

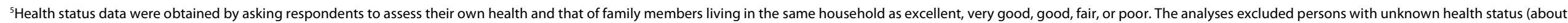
$0.1 \%)$.

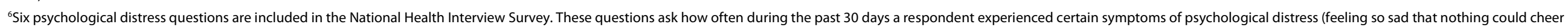

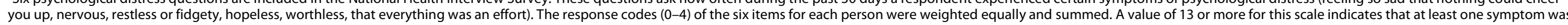
experienced "most of the time" or "all of the time" and is used here to define serious psychological distress. The analyses excluded adults with unknown serious psychological distress status (about 4\%).

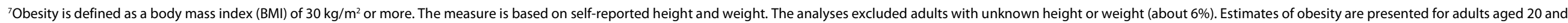
over because the Healthy People 2020 objectives (http://www.healthypeople.gov) for healthy weight among adults define adults as persons aged 20 and over.

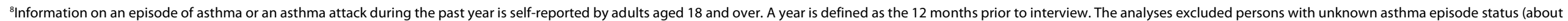
$0.1 \%)$.

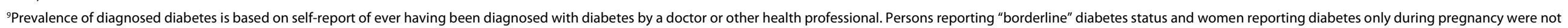
coded as having diabetes in the analyses. The analyses excluded adults with unknown diabetes status (about $0.1 \%$ ). 
Wireless Substitution: Early Release of Estimates From the National Health Interview Survey, July-December 2016

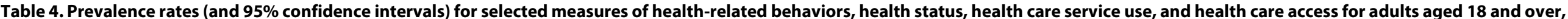
by household telephone status: United States, July-December 2016

Measure

Landline $^{1}$

Wireless-only

Phoneless

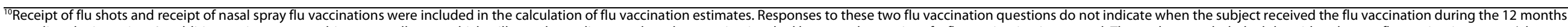

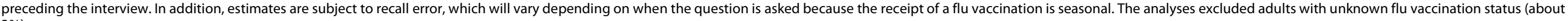
$3 \%$.

"IIndividuals who received human immunodeficiency virus (HIV) testing solely as a result of blood donation were considered not to have been tested for HIV. The analyses excluded adults with unknown HIV test status (about 5\%).

${ }^{12}$ Does not include a hospital emergency room. The analyses excluded persons with an unknown usual place to go for medical care (about 1.5\%).

${ }^{13} \mathrm{~A}$ year is defined as the 12 months prior to interview. The analyses excluded persons with unknown responses to the question on failure to obtain needed medical care due to cost (about $0.2 \%$ )

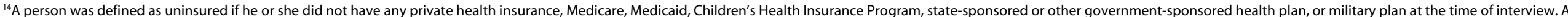

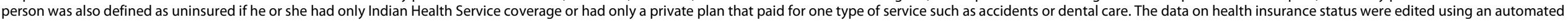
system based on logic checks and keyword searches. The analyses excluded adults with unknown health insurance status (about 1\%).

NOTE: Data are based on household interviews of a sample of the civilian noninstitutionalized population.

DATA SOURCE: CDC/NCHS, National Health Interview Survey, July-December 2016. 
Wireless Substitution: Early Release of Estimates From the National Health Interview Survey, July-December 2016

Table 5. Percentage of adults living in wireless-mostly households, by selected demographic characteristics and calendar half-years: United States, July $2013-D e c e m b e r ~ 2016$

\begin{tabular}{|c|c|c|c|c|c|c|c|c|}
\hline Demographic characteristic & $\begin{array}{l}\text { July-December } \\
2013\end{array}$ & $\begin{array}{c}\text { January-June } \\
2014\end{array}$ & $\begin{array}{l}\text { July-December } \\
2014\end{array}$ & $\begin{array}{c}\text { January-June } \\
2015\end{array}$ & $\begin{array}{l}\text { July-December } \\
2015\end{array}$ & $\begin{array}{c}\text { January-June } \\
2016\end{array}$ & $\begin{array}{l}\text { July-December } \\
2016\end{array}$ & $\begin{array}{l}95 \% \text { confidence } \\
\text { interval }^{1}\end{array}$ \\
\hline Total & 18.3 & 16.6 & 16.9 & 16.3 & 16.1 & 16.6 & 16.7 & $15.95-17.54$ \\
\hline \multicolumn{9}{|l|}{ Race/ethnicity } \\
\hline Hispanic or Latino, any race(s) & 16.6 & 14.6 & 14.2 & 15.4 & 15.0 & 14.5 & 15.6 & $13.63-17.75$ \\
\hline Non-Hispanic white, single race & 18.6 & 16.8 & 17.2 & 16.0 & 16.0 & 16.6 & 16.5 & $15.45-17.57$ \\
\hline Non-Hispanic black, single race & 18.2 & 16.9 & 17.5 & 17.3 & 17.1 & 18.4 & 17.5 & $15.81-19.36$ \\
\hline Non-Hispanic Asian, single race & 20.4 & 19.5 & 19.4 & 18.4 & 19.7 & 18.7 & 21.8 & $17.28-27.10$ \\
\hline Non-Hispanic other, single race & 14.1 & 11.0 & $* 10.3$ & 18.0 & 12.8 & 13.6 & 16.0 & $11.02-22.65$ \\
\hline Non-Hispanic, multiple race & 16.9 & 16.3 & 17.0 & 17.8 & 15.0 & 16.8 & 14.0 & $9.87-19.56$ \\
\hline \multicolumn{9}{|l|}{ Age (years) } \\
\hline $18-24$ & 20.0 & 18.1 & 17.7 & 17.1 & 17.2 & 16.5 & 17.2 & $15.43-19.14$ \\
\hline $25-29$ & 14.5 & 11.8 & 13.5 & 11.1 & 11.1 & 12.6 & 11.7 & $10.06-13.65$ \\
\hline $30-44$ & 20.0 & 17.6 & 17.2 & 16.9 & 16.2 & 16.5 & 15.9 & 14.79-16.99 \\
\hline $45-64$ & 21.6 & 20.0 & 20.6 & 19.2 & 19.9 & 20.1 & 20.7 & $19.62-21.86$ \\
\hline 65 and over & 10.3 & 10.2 & 10.6 & 12.0 & 11.0 & 12.5 & 12.9 & $11.99-13.95$ \\
\hline \multicolumn{9}{|l|}{ Sex } \\
\hline Male & 18.6 & 16.7 & 17.1 & 16.5 & 16.2 & 16.8 & 16.9 & $16.03-17.85$ \\
\hline Female & 18.0 & 16.5 & 16.7 & 16.1 & 16.1 & 16.4 & 16.6 & $15.79-17.35$ \\
\hline \multicolumn{9}{|l|}{ Education } \\
\hline Some high school or less & 12.4 & 12.4 & 11.0 & 12.1 & 12.1 & 12.8 & 12.2 & $10.92-13.62$ \\
\hline High school graduate or GED ${ }^{2}$ & 16.5 & 14.3 & 14.5 & 14.6 & 14.9 & 14.6 & 14.8 & $13.82-15.93$ \\
\hline Some post-high school, no degree & 18.9 & 17.3 & 17.7 & 16.4 & 15.8 & 16.9 & 17.4 & $16.25-18.51$ \\
\hline 4-year college degree or higher & 22.3 & 20.1 & 20.8 & 19.5 & 19.5 & 19.7 & 19.6 & $18.38-20.89$ \\
\hline \multicolumn{9}{|l|}{ Employment status last week } \\
\hline Working at a job or business & 21.4 & 18.9 & 19.5 & 18.2 & 18.3 & 18.0 & 18.4 & $17.47-19.35$ \\
\hline Keeping house & 16.9 & 15.9 & 16.8 & 13.9 & 15.5 & 15.7 & 16.9 & $14.83-19.30$ \\
\hline Going to school & 21.1 & 20.5 & 19.0 & 21.6 & 19.7 & 20.8 & 18.3 & $15.43-21.50$ \\
\hline Something else (incl. unemployed) & 11.4 & 11.2 & 10.9 & 11.9 & 11.4 & 13.2 & 13.0 & $12.17-13.87$ \\
\hline \multicolumn{9}{|l|}{ Household structure } \\
\hline Adult living alone & 9.4 & 9.3 & 9.3 & 9.5 & 9.5 & 10.1 & 9.9 & $9.00-10.80$ \\
\hline Unrelated adults, no children & 11.2 & 9.2 & 5.5 & 7.4 & *10.3 & 9.3 & *6.0 & $3.09-11.27$ \\
\hline Related adults, no children & 18.1 & 15.9 & 17.3 & 16.4 & 16.3 & 16.3 & 17.1 & $16.07-18.24$ \\
\hline Adult(s) with children & 22.6 & 20.8 & 20.0 & 19.2 & 19.2 & 20.0 & 19.4 & $18.17-20.68$ \\
\hline \multicolumn{9}{|l|}{ Household poverty status ${ }^{3}$} \\
\hline Poor & 9.1 & 9.1 & 8.4 & 10.0 & 8.7 & 9.7 & 10.0 & $8.45-11.82$ \\
\hline Near-poor & 12.0 & 10.6 & 12.0 & 12.5 & 10.7 & 12.8 & 11.1 & $9.43-12.91$ \\
\hline Not-poor & 22.1 & 20.0 & 19.4 & 18.4 & 18.7 & 18.6 & 18.9 & $17.85-19.96$ \\
\hline
\end{tabular}

See footnotes at end of table. 
Wireless Substitution: Early Release of Estimates From the National Health Interview Survey, July-December 2016

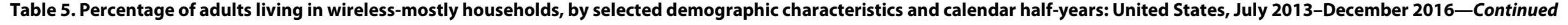

\begin{tabular}{|c|c|c|c|c|c|c|c|c|}
\hline Demographic characteristic & $\begin{array}{l}\text { July-December } \\
2013\end{array}$ & $\begin{array}{c}\text { January-June } \\
2014\end{array}$ & $\begin{array}{l}\text { July-December } \\
2014\end{array}$ & $\begin{array}{c}\text { January-June } \\
2015\end{array}$ & $\begin{array}{l}\text { July-December } \\
2015\end{array}$ & $\begin{array}{c}\text { January-June } \\
2016\end{array}$ & $\begin{array}{l}\text { July-December } \\
2016\end{array}$ & $\begin{array}{l}\text { 95\% confidence } \\
\text { interval }^{1}\end{array}$ \\
\hline \multicolumn{9}{|l|}{ Geographic region ${ }^{4}$} \\
\hline Northeast & 20.1 & 18.7 & 21.4 & 20.4 & 19.0 & 20.9 & 21.4 & $19.37-23.48$ \\
\hline Midwest & 16.2 & 14.5 & 14.6 & 13.1 & 14.9 & 13.9 & 15.0 & $13.34-16.91$ \\
\hline South & 18.0 & 16.0 & 16.2 & 16.3 & 15.6 & 16.0 & 15.8 & $14.61-16.99$ \\
\hline West & 19.3 & 18.1 & 16.5 & 16.2 & 15.9 & 16.7 & 16.2 & $14.50-17.94$ \\
\hline \multicolumn{9}{|l|}{ Metropolitan statistical area status } \\
\hline Metropolitan & 18.7 & 16.9 & 17.0 & 16.8 & 16.3 & 16.6 & 17.2 & $16.22-18.25$ \\
\hline Not metropolitan & 16.7 & 15.5 & 16.2 & 14.2 & 15.0 & 12.9 & 12.9 & $11.65-14.22$ \\
\hline \multicolumn{9}{|l|}{ Home ownership status ${ }^{5}$} \\
\hline Owned or being bought & 21.0 & 19.0 & 19.9 & 19.0 & 19.0 & 19.0 & 19.7 & $18.67-20.78$ \\
\hline Renting & 12.4 & 11.1 & 11.0 & 10.5 & 10.4 & 11.5 & 10.5 & $9.54-11.48$ \\
\hline Other arrangement & 14.8 & 12.8 & 12.1 & 14.1 & 11.7 & 16.3 & 14.9 & $10.94-20.06$ \\
\hline $\begin{array}{l}\text { Number of adults in survey sample } \\
\text { who live in landline households with } \\
\text { wireless telephones (unweighted) }\end{array}$ & 22,879 & 19,608 & 18,040 & 17,527 & 15,780 & 15,487 & 15,173 & $\ldots$ \\
\hline
\end{tabular}

* Estimate has a relative standard error greater than $30 \%$ and does not meet standards for reliability or precision.

... Category not applicable.

'Refers to July-December 2016

${ }^{2} \mathrm{GED}$ is General Educational Development high school equivalency diploma.

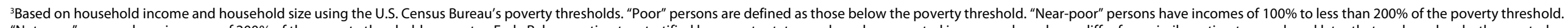

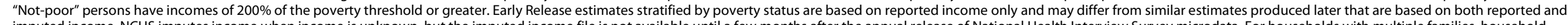

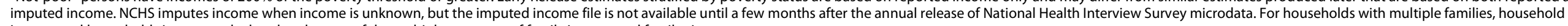
income and household size were calculated as the sum of the multiple measures of family income and family size.

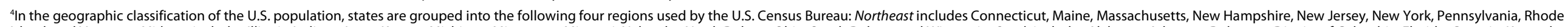

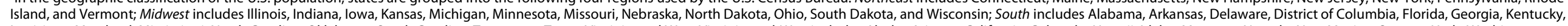

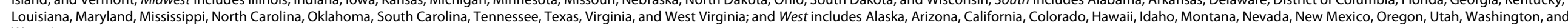
Wyoming.

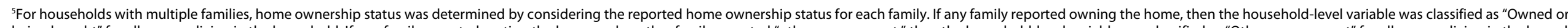

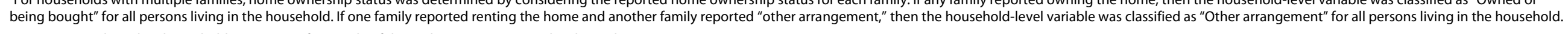
NOTE: Data are based on household interviews of a sample of the civilian noninstitutionalized population.

DATA SOURCE: CDC/NCHS, National Health Interview Survey, July 2013-December 2016. 